\title{
Is your prescribing serving a hidden addiction?
}

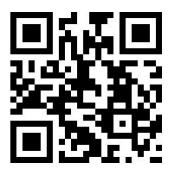

Addiction to prescription and over-the-counter (OTC) medicines is a hidden, little-researched chronic illness - which could be significantly countered by destigmatisation, 'up-scheduling' certain medications, creating a single electronic database across dispensaries and boosting doctor awareness regarding long-term or repeat prescriptions. This is according to Dr Volker Hitzeroth, President of the South African Addiction Medicine Society and convenor of the addictions sub-group of the South African Society of Psychiatrists.

Hitzeroth says low doctor awareness and sparse use of alternatives to addictive painkilling opioids add significantly to addiction. Since the only indication of the problem's spread and depth nationally is a dated (2003) study of 23 specialist substance abuse treatment centres in Cape Town, ${ }^{[1]}$ addiction treatment specialists have to lean heavily on an alarming 2012 United Nations World Drug report. It says that worldwide, up to 36 million people are dependent on opioids, nearly half of those seeking treatment for prescription medicine dependence reporting the use of opioid-containing painkillers. This latter estimate is extrapolated to South Africa and used as a basis for designing holistic countermeasures.

\section{Suspected prevalence: 'massive'}

Researchers and members of the South African Community Epidemiology Network on Drug Abuse (SACENDU) found that over two years (1998 to 2000) $7.8 \%$ of the 9063 cases recorded by specialist treatment centres in Cape Town reported OTC, prescription or 'unspecified' medicines as the primary or secondary substance of abuse.

'These are just the ones we know about,' said Hitzeroth. 'It's a very grey area - by the nature of these enquiries people hide information, it's difficult to own up; they don't want to or don't know it's a problem. The whole field is clouded in mystery ... but we suspect we actually have a massive prevalence.'

While the SACENDU study discovered benzodiazepines to be the most widely misused medicines in Cape Town (consistent with USA and European studies) and that more (middle-aged, higher income) women than men reported them as their primary substance of abuse, its authors question whether findings derived from a treatment context reflect medicine misuse in the general population. Like Hitzeroth, they speculate that OTC and prescription medicine abuse is 'far greater than we have observed'. Hitzeroth sees a wide range of OTC and prescription medicine addicts (including several healthcare professionals).

He says that in spite of the guidelines and regulations surrounding the prescription of pain medicines, and the accepted wisdom 


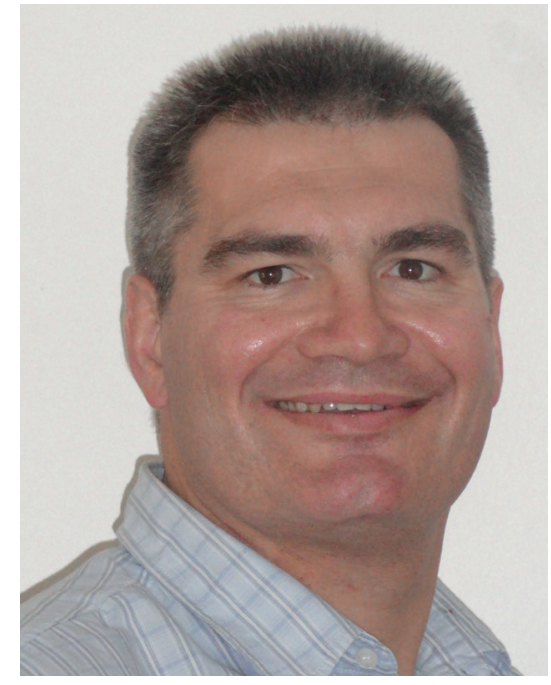

Dr Volker Hitzeroth, President of the South African Addiction Medicine Society.

of 'prescribing short-term, at lower doses', doctors continue to give three- and sixmonth scripts for Adcodol, Syndol, Myprodol and their associated generics all of which contain codeine - and for sleeping tablets that use benzodiazepines (particularly alprazolam).

\section{Mitigation and alternatives}

Hitzeroth is registered in the United Kingdom as a psychiatrist with a subspecialty in addictions (currently no such specialty is available at the HPCSA). $\mathrm{He}$ lectures in the subject and has written a book, The End of Addiction.

'The primary problem in this particular field is lack of awareness', he says. 'Doctors, pharmacists and patients are generally not very aware. When I see patients and families and ask if they knew the medication was addictive, they all say no. Then they're surprised that the drug has been prescribed non-stop for three years by various doctors.' Hitzeroth says doctors are under pressure to get patients better quickly and have limited time with each patient, while patients are demanding and pharmacists are under 'business pressure'. 'I've interviewed pharmacists who say if they don't give Adcodol the guy next door will', he adds.

One mitigating action would be to limit the availability of the 'known suspect' medicines via smaller packs and lower dosages, as well as up-scheduling the most abused drugs. A 'really useful' intervention would be to set up an electronic database, enabling all pharmacists and dispensing doctors/nurses to instantly view any patient's purchasing history. With patient consent, doctors in a group practice (including locums) could also share information about a single patient, thus avoiding becoming unwitting participants in a patient's drug habit. Hitzeroth said there are 'probably a handful of rotten doctors not doing the right thing and they need to be dealt with. If a patient or family are concerned they should report them with alacrity.'

Asked to give alternatives to prescription sleeping tablets, he listed antihistamines, certain sedating anti-depressants, a selection of low dose anti-psychotic medicines and a wide range of herbal remedies such as valerian and melatonin. Nonpharmacological management, such as sleep hygiene, is seldom used: 'You establish a bedtime routine, don't exercise, drink alcohol or smoke before sleeping and avoid daytime sleeps,' Hitzeroth explained. You use your bed only for sleeping and sexual recreation - not for watching TV, listening to music, playing DVD games or kids playing. A bedroom needs to be a calm, relaxing, quiet, pleasant environment with a comfortable temperature.'

\section{'The whole field is clouded in mystery ... but we suspect we actually have a massive prevalence, says Hitzeroth.}

\section{Lessons from overseas}

Hitzeroth says working overseas opened his eyes. 'We're far behind the rest of the world in terms of our approach to the treatment of addictions. I want to put addiction and treatment on the agenda, teach people. We also have a lot of cowboys in South Africa, less knowledgeable practitioners whose hearts are in the right place but who intervene in the wrong way, giving wrong prescriptions - and we're not up to scratch with the latest evidence base.'

The modern approach is to see addiction as a neuro-biological disease, not dissimilar to chronic relapsing illnesses like HIV or diabetes. He said one recent study ${ }^{[2]}$ revealed that outcomes for addictions were no worse than for other chronic illnesses. 'Yet everybody stigmatises addiction. We must treat it as a biopsychosocial illness, like all the others, and involve psychologists, social workers - whoever is needed for a holistic approach.'

He said pointers to addiction include repeated requests for specific codeinecontaining drugs, complaints of constant ongoing, 'unrelieved' pain (most abused medicines are for acute pain), stress and anxiety, and 'doctor-shopping and pharmacy-hopping' (different pharmacists being presented with different scripts for the same or similar medicine from different doctors). 'I've also had three cases where pharmacists actually picked up my forged signature and called me,' Hitzeroth warned.

\section{Interventions actually work}

The biggest upside, however, is that unlike those addicted to heroin, 'tik' or crack cocaine, OTC and prescription medicinedependent patients are more ready to quit, and respond 'very well' once a good doctor-patient rapport is established and some motivational work begun. 'They are very grateful that we can get them off,' says Hitzeroth. 'If they're really motivated we can get them off within a matter of weeks, occasionally months, depending on dosages and the meds involved. I have a whole range of patients I'm doing this with. It doesn't need to be a black hole that doctors must avoid and don't know how to manage. The core message is awareness: Ask the question (of the patient), engage, educate and motivate!'

Hitzeroth confirmed the SACENDU research profile of this category of drugdependent persons (OTC/PM): traditional middle to upper class, living in 'leafy suburbs'. However, 'We're seeing more and more professional, qualified working people and more with tertiary education, and, sadly, the previously underprivileged.' $\mathrm{He}$ explained that because OTCs and prescription medicines are so much cheaper than illicit drugs, even people who can't afford a consultation with a private doctor get them over the counter. A stereotypical profile of an OTC/PM-dependent person would be a middle-aged, successful white woman (with other groups fast catching up), perhaps also suffering pain, depression and/ or anxiety.

\section{Doctors continue to give three- month and six-month scripts for Adcodol, Syndol, Myprodol and their associated generics - all of which contain codeine - and for sleeping tablets that use benzodiazepines}

Hitzeroth has reassuring words for medical professionals with drug habits, many of whom are self-prescribing and believe themselves to be 'in control.' 'Yes, the HPCSA guidelines say colleagues are 
obliged to report them, but the overall purpose is not to deregister them but to treat them and keep them in the profession. My experience is they don't need to fear for their licences if they co-operate. The best route is to self-report and get help from the Medical Protection Society or similar outfit
- often it's marital, financial or work-related problems and lives start falling apart. The first step is to get rid of what's being used to numb the (emotional) pain.'

\section{Chris Bateman}

chrisb@hmpg.co.za
1. Myers B, Siegfried N, Parry C. Over-the-counter and prescription medicine misuse in Cape Town - findings from specialist treatment centres. S Afr Med J 2003;93(5):367-370.

2. McLellan TA, Lewis DC, O'Brein P, et al. Drug dependance, chronic medical illness: implications for treatment, insurance, and outcomes evaluation. JAMA 2000:284(13):1689-1695. http://dx.doi.org/10.1001/jama.284.13.1689]

S Afr Med J 2013;103(6):359-361.

DOI:10.7196/SAMJ.7027 